H. UEDA

KODAI MATH. J.

21 (1998), 273-284

\title{
MEROMORPHIC FUNCTIONS $f$ AND $g$ THAT SHARE TWO VALUES CM AND TWO OTHER VALUES IN THE SENSE OF \\ $E_{k}(\beta, f)=E_{k}(\beta, g)$
}

HidehaRu Ueda

\section{Introduction}

In this paper the term "meromorphic function" will mean a meromorphic function in $C$. We will use the standard notations of Nevanlinna theory: $T(r, f)$, $S(r, f), m(r, \beta, f), N(r, \beta, f), \bar{N}(r, \beta, f), N_{1}(r, \beta, f), \bar{N}_{1}(r, \beta, f), N_{1}(r, f), \bar{N}_{1}(r, f)$, $\Theta(\beta, f)(\beta \in C \cup\{\infty\}), \ldots$ etc., and we assume that the reader is familiar with the basic results in Nevanlinna theory as found in [3].

For a nonconstant meromorphic function $f$, a number $\beta \in C \cup\{\infty\}$ and a positive integer or $+\infty k$, we write $E_{k}(\beta, f)=\{z \in C: z$ is a $\beta$-point of $f$ with multiplicity less than or equal to $k$.\}.

If two nonconstant meromorphic functions $f$ and $g$ satisfy $E_{+\infty}(\beta, f)=$ $E_{+\infty}(\beta, g)$, then we say that $f$ and $g$ share $\beta I M$. If $f$ and $g$ satisfy $E_{k}(\beta, f)=$ $E_{k}(\beta, g)$ for all positive integers $k$, then we say that $f$ and $g$ share $\beta C M$. [1]:

The following Theorems A-C are due to Bhoosnurmath and Gopalakrishna

THEOREM A. Let $f$ and $g$ be nonconstant meromorphic functions. Assume that there exist distinct 5 elements $a_{1}, \ldots, a_{5}$ in $C \cup\{\infty\}$ such that $E_{k}\left(a_{j}, f\right)=$ $E_{k}\left(a_{j}, g\right)$ for $j=1, \ldots, 5$, where $k(\geqq 3)$ is a positive integer or $+\infty$. Then $f \equiv g$.

THEOREM B. Let $f$ and $g$ be nonconstant meromorphic functions. Assume that there exist distinct 6 elements $a_{1}, \ldots, a_{6}$ in $C \cup\{\infty\}$ such that $E_{2}\left(a_{j}, f\right)=$ $E_{2}\left(a_{j}, g\right)$ for $j=1, \ldots, 6$. Then $f \equiv g$.

THEOREM C. Let $f$ and $g$ be nonconstant meromorphic functions. Assume that there exist distinct 7 elements $a_{1}, \ldots, a_{7}$ in $C \cup\{\infty\}$ such that $E_{1}\left(a_{j}, f\right)=$ $E_{1}\left(a_{j}, g\right)$ for $j=1, \ldots, 7$. Then $f \equiv g$.

The case of $k=+\infty$ in Theorem $\mathrm{A}$ is a well-known result of Nevanlinna what is called Five-Point Theorem [5]. As we have pointed out in [6, p. 458], in

1991 Mathematics Subject Classification. Primary 30D35.

Key words and phrases: meromorphic functions, share $\mathrm{CM}, E_{k}(\beta, f)=E_{k}(\beta, g)$.

Received September 30, 1997; revised July 16, 1998. 
the above three results, the assumption on the number of distinct elements $\left\{a_{j}\right\}$ satisfying $E_{k}\left(a_{j}, f\right)=E_{k}\left(a_{j}, g\right)$ cannot be improved.

In connection with Theorems $B$ and $C$ we showed in [7] the following Theorems $\mathrm{D}$ and $\mathrm{E}$.

THEOREM D. Let $f$ and $g$ be nonconstant meromorphic functions. Assume that $f$ and $g$ share two values 0 and $\infty C M$, and further that they satisfy $E_{2}\left(a_{j}, f\right)=$ $E_{2}\left(a_{j}, g\right)$ for $j=3,4,5$, where $a_{3}=1, a_{4}=a, a_{5}=b .(a, b \neq 0, \infty, 1 ; a \neq b)$ (i) If $\{a, b\}=\left\{\omega, \omega^{2}\right\}$, where $\omega(\neq 1)$ is a third root of unity, then $f^{3} \equiv g^{3}$. If $\{a, b\} \neq\left\{\omega, \omega^{2}\right\}$, then $f \equiv g$.

THEOREM E. Let $f$ and $g$ be nonconstant meromorphic functions. Assume that $f$ and $g$ share two values 0 and $\infty C M$, and further that they satisfy $E_{1}\left(a_{j}, f\right)=$ $E_{1}\left(a_{j}, g\right)$ for $j=3, \ldots, 6$, where $a_{3}=1, a_{4}=a, a_{5}=b, a_{6}=c .(a, b, c \neq 0, \infty, 1$; $a \neq b \neq c \neq a)$ (i) If $\{a, b, c\}=\{i,-1,-i\}$, then $f^{4} \equiv g^{4}$. (ii) If $\{a, b, c\}=\{\alpha$, $-1,-\alpha\}(\alpha \neq \pm i)$, then $f^{2} \equiv g^{2}$. (iii) If $\{a, b, c\} \neq\{\alpha,-1,-\alpha\}$, then $f \equiv g$.

Gundersen [2] proved the following result which generalizes a well-known result of Nevanlinna what is called Four-Point Theorem [5].

THEOREM F. Let $f$ and $g$ be nonconstant meromorphic functions. Assume that $f$ and $g$ share two values 0 and $\infty C M$, and that they share two values 1 and $a(\neq 0, \infty, 1) I M$. (i) If $a=-1$, then $f g \equiv 1, f+g \equiv 0$ or $f \equiv g$. (ii) If $a=1 / 2$, then $(f-(1 / 2))(g-(1 / 2)) \equiv 1 / 4, f+g \equiv 1$ or $f \equiv g$. (iii) If $a=2$, then $(f-1)(g-1) \equiv 1, f+g \equiv 2$ or $f \equiv g$. (iv) If $a \neq-1,1 / 2,2$, then $f \equiv g$. results.

In this paper in relation to Theorems $\mathrm{A}$ and $\mathrm{F}$ we prove the following two

THEOREM 1. Let $f$ and $g$ be nonconstant meromorphic functions. Assume that $f$ and $g$ share two values 0 and $\infty C M$, and that they satisfy $E_{k}\left(a_{j}, f\right)=E_{k}\left(a_{j}, g\right)$ for $j=3,4$, where $a_{3}=1, a_{4}=a(\neq 0, \infty, 1,-1)$ and $k(\geqq 12)$ is a positive integer. (i) If $a=1 / 2$, then $(f-(1 / 2))(g-(1 / 2)) \equiv 1 / 4, f+g \equiv 1$ or $f \equiv g$. (ii) If $a=2$, then $(f-1)(g-1) \equiv 1, f+g \equiv 2$ or $f \equiv g$. (iii) If $a \neq-1,1 / 2,2$, then $f \equiv g$.

THEOREM 2. Let $f$ and $g$ be nonconstant meromorphic functions. Assume that $f$ and $g$ share two values 0 and $\infty C M$, and that they satisfy $E_{k}\left(a_{j}, f\right)=$ $E_{k}\left(a_{j}, g\right)$ for $j=3,4$, where $a_{3}=1, a_{4}=-1$ and $k(\geqq 7)$ is a positive integer. Then $f g \equiv 1, f+g \equiv 0$ or $f \equiv g$.

\section{Notations and terminology}

In this section, we introduce some notations and terminology which will be needed to prove Theorems 1 and 2 . 
〈i Let $f, g$ be distinct nonconstant meromorphic functions. For $r>0$, put $T(r)=\max \{T(r, f), T(r, g)\}$. We write $\sigma(r)=S(r)$ for every function $\sigma:(0,+\infty) \rightarrow$ $(-\infty,+\infty)$ satisfying $\sigma(r) / T(r) \rightarrow 0$ for $r \rightarrow+\infty$ possibly outside a set of finite Lebesgue measure.

〈ii) Let $f, g$ be nonconstant meromorphic functions. We denote by $\bar{N}_{c}(r, \beta ; f, g) \equiv \bar{N}_{c}(r, \beta)$ (resp. $\left.\bar{N}_{d}(r, \beta ; f, g) \equiv \bar{N}_{d}(r, \beta)\right)$ the counting function of those common $\beta$-points of $f$ and $g$ with the same multiplicity (resp. with the different multiplicities), each point counted only once regardless of multiplicity, and we write $\bar{N}_{i}(r, \beta ; f, g) \equiv \bar{N}_{i}(r, \beta)=\bar{N}_{c}(r, \beta)+\bar{N}_{d}(r, \beta)$.

We say that $f$ and $g$ share $\beta C M^{\prime \prime}$ if $\bar{N}(r, \beta, f)-\bar{N}_{c}(r, \beta)=S(r, f)$ and $\bar{N}(r, \beta, g)-\bar{N}_{c}(r, \beta)=S(r, g)$ hold. Similarly, if $\bar{N}(r, \beta, f)-\bar{N}_{i}(r, \beta)=S(r, f)$ and $\bar{N}(r, \beta, g)-\bar{N}_{i}(r, \beta)=S(r, g)$ hold, then we say that $f$ and $g$ share $\beta I M^{\prime \prime}$. These notions $\mathrm{CM}^{\prime \prime}$ and $\mathrm{IM}^{\prime \prime}$ are slight generalizations of $\mathrm{CM}$ and IM, respectively.

〈iii) Let $f$ and $g$ be nonconstant meromorphic functions. For $\beta, \gamma$ $(\in C \cup\{\infty\}), \beta \neq \gamma$ we put

$$
\begin{gathered}
m_{\beta, \gamma}(r) \equiv m_{\beta, \gamma}(r ; f, g)=m(r, \beta, f)+m(r, \gamma, f)+m(r, \beta, g)+m(r, \gamma, g), \\
\bar{N}_{\beta, \gamma}(r) \equiv \bar{N}_{\beta, \gamma}(r ; f, g)=\bar{N}(r ; f=\beta, g \neq \beta)+\bar{N}(r ; f=\gamma, g \neq \gamma) \\
+\bar{N}(r ; g=\beta, f \neq \beta)+\bar{N}(r ; g=\gamma, f \neq \gamma), \\
\tilde{N}_{\beta, \gamma}^{\prime}(r) \equiv \tilde{N}_{\beta, \gamma}^{\prime}(r ; f, g)=\bar{N}_{c}(r, \beta)+\bar{N}_{c}(r, \gamma), \\
\tilde{N}_{\beta, \gamma}^{\prime \prime}(r) \equiv \tilde{N}_{\beta, \gamma}^{\prime \prime}(r ; f, g)=\bar{N}_{d}(r, \beta)+\bar{N}_{d}(r, \gamma), \\
\tilde{N}_{\beta, \gamma}(r) \equiv \tilde{N}_{\beta, \gamma}(r ; f, g)=\tilde{N}_{\beta, \gamma}^{\prime}(r ; f, g)+\tilde{N}_{\beta, \gamma}^{\prime \prime}(r ; f, g)=\bar{N}_{i}(r, \beta ; f, g)+\bar{N}_{i}(r, \gamma ; f, g),
\end{gathered}
$$

where for example, $\bar{N}(r ; f=\beta, g \neq \beta)$ denotes the counting function of those $\beta$ points of $f$ which are not $\beta$-points of $g$, each point counted only once.

\section{Preparations for the proof of Theorems 1 and 2}

We often need a slight generalization of Theorem F:

THEOREM $\mathrm{F}^{\prime}$. Theorem $F$ remains still valid if $C M$ and $I M$ are replaced by $C M^{\prime \prime}$ and $I M^{\prime \prime}$, respectively.

In order to prove this fact we have only to use the argument (due to Mues) of the proof of Theorem 1 in [4] by replacing CM and IM by $\mathrm{CM}^{\prime \prime}$ and $\mathrm{IM}^{\prime \prime}$, respectively.

In the rest of this section, we assume that $f$ and $g$ are distinct nonconstant meromorphic functions sharing $a_{1}=0$ and $a_{2}=\infty \mathrm{CM}$ and satisfying $E_{k}\left(a_{j}, f\right)=$ $E_{k}\left(a_{j}, g\right)$ for $j=3,4$, where $a_{3}=1, a_{4}=a(\neq 0, \infty, 1)$ and $k(\geqq 2)$ is a positive integer. We write, for example, $N(r, 0, f)=N(r, 0, g)=N(r, 0), N(r, \infty, f)=$ $N(r, \infty, g)=N(r, \infty), \bar{N}(r, 0, f)=\bar{N}(r, 0, g)=\bar{N}(r, 0), \bar{N}(r, \infty, f)=\bar{N}(r, \infty, g)=$ $\bar{N}(r, \infty), N_{1}(r, 0, f)=N_{1}(r, 0, g)=N_{1}(r, 0), N_{1}(r, \infty, f)=N_{1}(r, \infty, g)=N_{1}(r, \infty)$, $\bar{N}_{1}(r, 0, f)=\bar{N}_{1}(r, 0, g)=\bar{N}_{1}(r, 0), \bar{N}_{1}(r, \infty, f)=\bar{N}_{1}(r, \infty, g)=\bar{N}_{1}(r, \infty)$. 
LEMMA 1. $S(r)=S(r, f)=S(r, g)$.

Proof. Let $d \in C$ be different from $a_{j}(j=1,2,3,4)$, and let $b_{j}=\left(a_{j}-d\right)^{-1}$ $(j=1,2,3,4)$. Then $b_{1}, \ldots, b_{4}$ are all distinct and finite. If we put $F=(f-d)^{-1}$ and $G=(g-d)^{-1}$, then $F$ and $G$ share $b_{1}$ and $b_{2}$ CM and satisfy $E_{k}\left(b_{j}, F\right)=$ $E_{k}\left(b_{j}, G\right)$ for $j=3,4$. By the second fundamental theorem and the fact that $F \not \equiv G$

$$
\begin{aligned}
2 T(r, F) \leqq & \sum_{j=1}^{4} \bar{N}\left(r, b_{j}, F\right)+S(r, F) \\
\leqq & \sum_{j=1}^{2} \bar{N}\left(r, b_{j}, F\right)+\tilde{N}_{b_{3}, b_{4}}(r ; F, G) \\
& +\sum_{j=3}^{4} \bar{N}\left(r ; F=b_{j}, G \neq b_{j}\right)+S(r, F) \\
\leqq & N(r, 0, F-G)+\{2 /(k+1)\} T(r, F)+S(r, F) \\
\leqq & T(r, F)+T(r, G)+\{2 /(k+1)\} T(r, F)+S(r, F)
\end{aligned}
$$

i.e.,

$$
T(r, F) \leqq\{(k+1) /(k-1)\} T(r, G)+S(r, F) .
$$

(3.1) is still valid when we exchange $F$ and $G$, so that

$$
T(r, G) \leqq\{(k+1) /(k-1)\} T(r, F)+S(r, G) .
$$

Taking $T(r, F)=T(r, f)+O(1)$ and $T(r, G)=T(r, g)+O(1)$ into account, we immediately deduce Lemma 1 from (3.1) and (3.2).

Lemma 2. Let $\tilde{n}\left(r ; f^{\prime}=g^{\prime}=0, f \neq 0, g \neq 0\right)$ denote the number of distinct common zeros of $f^{\prime}$ and $g^{\prime}$ which are neither zeros of $f$ nor $g$ in $|z| \leqq r$. Put $\tilde{N}\left(r ; f^{\prime}=g^{\prime}=0, f \neq 0, g \neq 0\right)=\int_{0}^{r}\left\{\tilde{n}\left(t ; f^{\prime}=g^{\prime}=0, f \neq 0, g \neq 0\right)-\tilde{n}\left(0 ; f^{\prime}=g^{\prime}=0\right.\right.$, $f \neq 0, g \neq 0)\} / t d t+\tilde{n}\left(0 ; f^{\prime}=g^{\prime}=0, f \neq 0, g \neq 0\right) \log r$. If $g / f$ is not a constant, then $\tilde{N}\left(r ; f^{\prime}=g^{\prime}=0, f \neq 0, g \neq 0\right)=S(r)$.

Proof. Since $f$ and $g$ share 0 and $\infty \mathrm{CM}$, there is an entire function $\alpha$ satisfying $g=e^{\alpha} f$, where $\alpha$ is nonconstant. Assume that there is a point $z_{0}$ such that $f^{\prime}\left(z_{0}\right)=g^{\prime}\left(z_{0}\right)=0, f\left(z_{0}\right) \neq 0$ and $g\left(z_{0}\right) \neq 0$. The differentiation of $g=e^{\alpha} f$ gives $g^{\prime}=e^{\alpha}\left(\alpha^{\prime} f+f^{\prime}\right)$, and so we have $\alpha^{\prime}\left(z_{0}\right)=0$. Since $\alpha$ is entire, we deduce using the lemma of the logarithmic derivative that

$$
\begin{aligned}
\tilde{N}\left(r ; f^{\prime}=g^{\prime}=0, f \neq 0, g \neq 0\right) & \leqq \bar{N}\left(r, 0, \alpha^{\prime}\right) \leqq m\left(r, \alpha^{\prime}\right)+O(1) \\
& =m\left\{r,\left(e^{\alpha}\right)^{\prime} / e^{\alpha}\right\}+O(1)=S\left(r, e^{\alpha}\right) \\
& =S(r, g / f) \leqq S(r, f)+S(r, g)=S(r) .
\end{aligned}
$$


LEMMA 3. Let $n_{1}^{\prime}(r, f)$ denote the number of multiple points of $f$ in $|z| \leqq r$ such that $f \neq 0, \infty, 1, a$, where a point of multiplicity $m$ is counted $(m-1)$ times, and put $N_{1}^{\prime}(r, f)=\int_{0}^{r}\left\{n_{1}^{\prime}(t, f)-n_{1}^{\prime}(0, f)\right\} / t d t+n_{1}^{\prime}(0, f) \log r$. If $N_{1}^{\prime}(r, g)$ is similarly defined, then

$$
\begin{aligned}
& \tilde{N}_{1, a}^{\prime \prime}(r ; f, g)+k \bar{N}_{1, a}(r ; f, g)+N_{1}^{\prime}(r, f)+N_{1}^{\prime}(r, g) \\
& \leqq 2\{\bar{N}(r, 0)+\bar{N}(r, \infty)\}+S(r)
\end{aligned}
$$

Proof. By the first and the second fundamental theorems

$$
\begin{aligned}
& m_{1, a}(r ; f, g)+2 \tilde{N}_{1, a}^{\prime}(r ; f, g)+3 \tilde{N}_{1, a}^{\prime \prime}(r ; f, g)+(k+1) \bar{N}_{1, a}(r ; f, g) \\
& \leqq m_{1, a}(r ; f, g)+N(r, 1, f)+N(r, 1, g)+N(r, a, f)+N(r, a, g) \\
&= 2\{T(r, f)+T(r, g)\}+O(1) \\
& \leqq \sum_{J=1}^{4}\left\{\bar{N}\left(r, a_{j}, f\right)+\bar{N}\left(r, a_{j}, g\right)\right\}-\left\{N_{1}^{\prime}(r, f)+N_{1}^{\prime}(r, g)\right\}+S(r) \\
&= 2\left\{\bar{N}(r, 0)+\bar{N}(r, \infty)+\tilde{N}_{1, a}(r ; f, g)\right\}+\bar{N}_{1, a}(r ; f, g) \\
&-\left\{N_{1}^{\prime}(r, f)+N_{1}^{\prime}(r, g)\right\}+S(r),
\end{aligned}
$$

from which we immediately deduce (3.3).

Now, we introduce some auxiliary functions:

$$
\begin{gathered}
\phi_{1}=\frac{f^{\prime} g^{\prime}(f-g)^{2}}{f g(f-1)(g-1)(f-a)(g-a)} \quad(\not \equiv 0), \\
\phi_{2}=\frac{f^{\prime} f}{(f-1)(f-a)}-\frac{g^{\prime} g}{(g-1)(g-a)} \\
\phi_{3}=\frac{f^{\prime}}{f(f-1)(f-a)}-\frac{g^{\prime}}{g(g-1)(g-a)}, \\
\phi_{4}=\left(\frac{f^{\prime \prime}}{f^{\prime}}-2 \frac{f^{\prime}}{f}-\frac{f^{\prime}}{f-1}-\frac{f^{\prime}}{f-a}\right)-\left(\frac{g^{\prime \prime}}{g^{\prime}}-2 \frac{g^{\prime}}{g}-\frac{g^{\prime}}{g-1}-\frac{g^{\prime}}{g-a}\right), \\
\phi_{5}=\left(\frac{f^{\prime \prime}}{f^{\prime}}+2 \frac{f^{\prime}}{f}-\frac{f^{\prime}}{f-1}-\frac{f^{\prime}}{f-a}\right)-\left(\frac{g^{\prime \prime}}{g^{\prime}}+2 \frac{g^{\prime}}{g}-\frac{g^{\prime}}{g-1}-\frac{g^{\prime}}{g-a}\right), \\
\phi_{8}=\left(\frac{f^{\prime \prime}}{f^{\prime}}-2 \frac{f^{\prime}}{f}-\frac{f^{\prime}}{f-1}+\frac{a f^{\prime}}{f-a}\right)-\left(\frac{g^{\prime \prime}}{g^{\prime}}-2 \frac{g^{\prime}}{g}-\frac{g^{\prime}}{g-1}+\frac{a g^{\prime}}{g-a}\right),
\end{gathered}
$$


and

$$
\begin{aligned}
\phi_{9}= & \left(\frac{f^{\prime \prime}}{f^{\prime}}+(1-a) \frac{f^{\prime}}{f}+\frac{a f^{\prime}}{f-1}-\frac{f^{\prime}}{f-a}\right) \\
& -\left(\frac{g^{\prime \prime}}{g^{\prime}}+(1-a) \frac{g^{\prime}}{g}+\frac{a g^{\prime}}{g-1}-\frac{g^{\prime}}{g-a}\right) .
\end{aligned}
$$

We remark that for the case $a=-1, \phi_{8} \equiv \phi_{4}$ and $\phi_{9} \equiv \phi_{5}$ hold. With the aid of these auxiliary functions we obtain some basic estimates:

LEMMA 4. (i)

$$
2\left\{N_{1}(r, 0)+N_{1}(r, \infty)\right\}+\bar{N}_{1}^{\prime}(r, f)+\bar{N}_{1}^{\prime}(r, g) \leqq \bar{N}_{1, a}(r)+S(r) .
$$

(ii) If neither $\phi_{2} \equiv 0$ nor $\phi_{3} \equiv 0$, then

$$
\bar{N}(r, 0)+\bar{N}(r, \infty) \leqq 2\left\{\bar{N}_{1, a}(r)+\tilde{N}_{1, a}^{\prime \prime}(r)\right\}+S(r) .
$$

(iii) If neither $\phi_{6} \equiv 0$ nor $\phi_{7} \equiv 0$, then

$$
\bar{N}(r, 0)+\bar{N}(r, \infty)\left\{\begin{aligned}
\leqq & 4 \bar{N}_{1, a}(r)+4\left\{\bar{N}_{1}^{\prime}(r, f)+\bar{N}_{1}^{\prime}(r, g)\right\} \\
& +\bar{N}_{1}(r, 0)+\bar{N}_{1}(r, \infty)+S(r) \quad(a \neq-1), \\
\leqq & 2 \bar{N}_{1, a}(r)+2\left\{\bar{N}_{1}^{\prime}(r, f)+\bar{N}_{1}^{\prime}(r, g)\right\} \\
& +\bar{N}_{1}(r, 0)+\bar{N}_{1}(r, \infty)+S(r) \quad(a=-1),
\end{aligned}\right.
$$

where for example, $\bar{N}_{1}^{\prime}(r, f)$ denotes the counting function of multiple points of $f(\neq 0, \infty, 1, a)$, each point counted only once.

(iv) If neither $\phi_{8} \equiv 0$ nor $\phi_{9} \equiv 0$, then

$$
\begin{aligned}
\bar{N}(r, 0)+\bar{N}(r, \infty) \leqq & \tilde{N}_{1, a}^{\prime \prime}(r)+2 \bar{N}_{1, a}(r)+2\left\{\bar{N}_{1}^{\prime}(r, f)+\bar{N}_{1}^{\prime}(r, g)\right\} \\
& +\bar{N}_{1}(r, 0)+\bar{N}_{1}(r, \infty)+S(r) \quad(a \neq-1) .
\end{aligned}
$$

Proof. (i) From the fundamental estimate of the logarithmic derivative it follows that $m\left(r, \phi_{1}\right)=S(r)$ (cf. [4, p. 171]). The poles of $\phi_{1}$ occur with multiplicity 1 due to the case [i] the 1 - or $a$ - points of $f$ (resp. $g$ ) which are simple points of $g(\neq 1, a)$ (resp. $f(\neq 1, a)$ ), and with multiplicity 2 due to the case [ii] the common roots of $f=1$ (resp. $f=a$ ) and $g=a$ (resp. $g=1$ ). Hence we have $N\left(r, \infty, \phi_{1}\right)=\bar{N}_{1, a}(r)-\left\{\bar{N}_{1}^{\prime}(r, f ; g=1, a)+\bar{N}_{1}^{\prime}(r, g ; f=1, a)\right\}$, where for example, $\bar{N}_{1}^{\prime}(r, f ; g=1, a)$ denotes the counting function of those multiple points of $f(\neq 0, \infty, 1, a)$ which are either 1 - or $a$-points of $g$, each point counted only once. Since $\phi_{1} \not \equiv 0$, we obtain from the first fundamental theorem

$$
\begin{aligned}
& 2\left\{N_{1}(r, 0)+N_{1}(r, \infty)\right\}+\bar{N}_{1}^{\prime}(r, f ; g \neq 1, a)+\bar{N}_{1}^{\prime}(r, g ; f \neq 1, a) \\
& \quad \leqq N\left(r, 0, \phi_{1}\right) \leqq T\left(r, \phi_{1}\right)+O(1) \\
& \quad=\bar{N}_{1, a}(r)-\left\{\bar{N}_{1}^{\prime}(r, f ; g=1, a)+\bar{N}_{1}^{\prime}(r, g ; f=1, a)\right\}+S(r)
\end{aligned}
$$


where for example, $\bar{N}_{1}^{\prime}(r, f ; g \neq 1, a)$ denotes the counting function of those multiple points of $f(\neq 0, \infty, 1, a)$ which are neither 1 - nor $a$-points of $g$, each point counted only once. From $(3.13)^{\prime}$ we immediately deduce (3.13).

(ii) From our assumption that $\phi_{2} \not \equiv 0$ and $\phi_{3} \not \equiv 0$, it follows that

$$
\begin{aligned}
\bar{N}(r, 0) & \leqq N\left(r, 0, \phi_{2}\right) \leqq T\left(r, \phi_{2}\right)+O(1)=m\left(r, \phi_{2}\right)+N\left(r, \infty, \phi_{2}\right)+O(1) \\
& \leqq \bar{N}_{1, a}(r)+\tilde{N}_{1, a}^{\prime \prime}(r)+S(r)
\end{aligned}
$$

and

$$
\begin{aligned}
\bar{N}(r, \infty) & \leqq N\left(r, 0, \phi_{3}\right) \leqq T\left(r, \phi_{3}\right)+O(1)=m\left(r, \phi_{3}\right)+N\left(r, \infty, \phi_{3}\right)+O(1) \\
& \leqq \bar{N}_{1, a}(r)+\tilde{N}_{1, a}^{\prime \prime}(r)+S(r)
\end{aligned}
$$

Combining these inequalities we have (3.14).

(iii) Let $z_{0}$ be a common simple zero of $f$ and $g$. Then we easily see that $\phi_{6}\left(z_{0}\right)=0$. Hence our assumption $\phi_{6} \not \equiv 0$ gives

$$
\begin{aligned}
\bar{N}(r, 0) & \leqq N\left(r, 0, \phi_{6}\right)+\bar{N}_{1}(r, 0) \leqq T\left(r, \phi_{6}\right)+\bar{N}_{1}(r, 0)+O(1) \\
& =m\left(r, \phi_{6}\right)+N\left(r, \infty, \phi_{6}\right)+\bar{N}_{1}(r, 0)+O(1) \\
& =N\left(r, \infty, \phi_{6}\right)+\bar{N}_{1}(r, 0)+S(r) \\
& \leqq 2 \bar{N}_{1, a}(r)+2\left\{\bar{N}_{1}^{\prime}(r, f)+\bar{N}_{1}^{\prime}(r, g)\right\}+\bar{N}_{1}(r, 0)+S(r) .
\end{aligned}
$$

(In particular, if $a=-1$, then we obtain

$$
\begin{aligned}
\bar{N}(r, 0) & \leqq N\left(r, 0, \phi_{4}\right)+\bar{N}_{1}(r, 0) \leqq T\left(r, \phi_{4}\right)+\bar{N}_{1}(r, 0)+O(1) \\
& =N\left(r, \infty, \phi_{4}\right)+\bar{N}_{1}(r, 0)+S(r) \\
& \left.\leqq \bar{N}_{1, a}(r)+\bar{N}_{1}^{\prime}(r, f)+\bar{N}_{1}^{\prime}(r, g)+\bar{N}_{1}(r, 0)+S(r) .\right)
\end{aligned}
$$

Next, let $z_{\infty}$ be a common simple pole of $f$ and $g$. Then we have $\phi_{7}\left(z_{\infty}\right)=0$. Using the assumption that $\phi_{7} \not \equiv 0$, we obtain

$$
\begin{aligned}
\bar{N}(r, \infty) & \leqq N\left(r, 0, \phi_{7}\right)+\bar{N}_{1}(r, \infty) \leqq T\left(r, \phi_{7}\right)+\bar{N}_{1}(r, \infty)+O(1) \\
& =m\left(r, \phi_{7}\right)+N\left(r, \infty, \phi_{7}\right)+\bar{N}_{1}(r, \infty)+O(1) \\
& =N\left(r, \infty, \phi_{7}\right)+\bar{N}_{1}(r, \infty)+S(r) \\
& \leqq 2 \bar{N}_{1, a}(r)+2\left\{\bar{N}_{1}^{\prime}(r, f)+\bar{N}_{1}^{\prime}(r, g)\right\}+\bar{N}_{1}(r, \infty)+S(r) .
\end{aligned}
$$

(In particular, if $a=-1$, then we get 


$$
\begin{aligned}
\bar{N}(r, \infty) & \leqq N\left(r, 0, \phi_{5}\right)+\bar{N}_{1}(r, \infty) \leqq T\left(r, \phi_{5}\right)+\bar{N}_{1}(r, \infty)+O(1) \\
& =N\left(r, \infty, \phi_{5}\right)+\bar{N}_{1}(r, \infty)+S(r) \\
& \left.\leqq \bar{N}_{1, a}(r)+\left\{\bar{N}_{1}^{\prime}(r, f)+\bar{N}_{1}^{\prime}(r, g)\right\}+\bar{N}_{1}(r, \infty)+S(r) .\right)
\end{aligned}
$$

The combination of the above two estimates yields (3.15).

(iv) If $z_{0}$ (resp. $z_{\infty}$ ) is a common simple zero (resp. pole) of $f$ and $g$, then $\phi_{8}\left(z_{0}\right)=0$ (resp. $\phi_{9}\left(z_{\infty}\right)=0$ ). Since we assume that $\phi_{8} \not \equiv 0$ and $\phi_{9} \not \equiv 0$, we easily see that

$$
\begin{aligned}
\bar{N}(r, 0)+\bar{N}(r, \infty) \leqq & N\left(r, 0, \phi_{8}\right)+N\left(r, 0, \phi_{9}\right)+\bar{N}_{1}(r, 0)+\bar{N}_{1}(r, \infty) \\
\leqq & N\left(r, \infty, \phi_{8}\right)+N\left(r, \infty, \phi_{9}\right)+\bar{N}_{1}(r, 0)+\bar{N}_{1}(r, \infty)+S(r) \\
\leqq & \tilde{N}_{1, a}^{\prime \prime}(r)+2 \bar{N}_{1, a}(r)+2\left\{\bar{N}_{1}^{\prime}(r, f)+\bar{N}_{1}^{\prime}(r, g)\right\} \\
& +\bar{N}_{1}(r, 0)+\bar{N}_{1}(r, \infty)+S(r) .
\end{aligned}
$$

\section{Proof of Theorems 1 and 2}

In what follows we assume that $f$ and $g$ are distinct and satisfy the assumptions of Theorem 1 or 2 , and so there is an entire function $\alpha$ satisfying $g=e^{\alpha} f\left(e^{\alpha} \not \equiv 1\right)$.

CASE 1. We first consider the case that $e^{\alpha}$ is a constant $C(\neq 0,1)$. From the assumptions $E_{k}(1, f)=E_{k}(1, g)$ and $E_{k}(a, f)=E_{k}(a, g)$ it follows that $\Theta(1, g)$, $\Theta(a, g) \geqq k /(k+1)$. If $C \neq a$, we also obtain $\Theta(C, g) \geqq k /(k+1)$, and so $\Theta(1, g)+$ $\Theta(a, g)+\Theta(C, g) \geqq 3 k /(k+1)>2$, a contradiction. This shows $C=a$. Further if $a^{2} \neq 1$, we also obtain $\Theta\left(a^{2}, g\right) \geqq k /(k+1)$, and so $\Theta(1, g)+\Theta(a, g)+\Theta\left(a^{2}, g\right)$ $\geqq 3 k /(k+1)>2$, a contradiction. This shows $a^{2}=1$, i.e., $a=-1$ and $f+g \equiv 0$. In this case we remark that $N(r, 1, f)=N(r,-1, g)$ and $N(r,-1, f)=N(r, 1, g)$ are not necessarily $S(r)$ !

CASE 2. We next consider the case that $e^{\alpha}$ is nonconstant. We divide our argument into several subcases:

2.1. The case $\phi_{2} \equiv 0$

$\phi_{2} \equiv 0$ implies that any 1- and $a$-point of $f$ (resp. $g$ ) is a 1- or an $a$-point of $g$ (resp. $f$ ). By making use of Lemma 2, we deduce from the assumptions $E_{k}\left(a_{j}, f\right)=E_{k}\left(a_{j}, g\right)$ for $j=3,4$ with $a_{3}=1, a_{4}=a$ that $\bar{N}(r ; f=1, g=a)+$ $\bar{N}(r ; f=a, g=1)=S(r)$, (where $\bar{N}(r ; f=1, g=a)$ denotes the counting function of common roots of $f=1$ and $g=a$, each counted only once,) and so by Lemma $1 f$ and $g$ share two values 1 and $a$ IM $^{\prime \prime}$. Hence by Theorem $\mathrm{F}^{\prime} f$ and $g$ are connected with one of the relations stated in Theorem F. Further, 
straightforward computations show that only two relations $(f-(1 / 2))$ $(g-(1 / 2)) \equiv 1 / 4$ (with $a=1 / 2)$ and $(f-1)(g-1) \equiv 1$ (with $a=2$ ) are suitable for $\phi_{2} \equiv 0$.

\subsection{The case $\phi_{3} \equiv 0$}

The same reasoning as in the case 2.1 shows that only two relations $f+g \equiv 2$ (with $a=2$ ) and $f+g \equiv 1$ (with $a=1 / 2$ ) are suitable for $\phi_{3} \equiv 0$.

2.3. The case $\phi_{6} \equiv 0$

First we consider the case $a \neq-1$. By (3.9)

$$
\phi_{4}^{2} \equiv(1+a)^{2} \phi_{1} \text {. }
$$

The poles of the right hand side of (4.1) occur with multiplicity 1 due to the case [i] the 1- or $a$-points of $f$ (resp. $g$ ) which are simple points of $g(\neq 1, a)$ (resp. $f(\neq 1, a)$ ), and with multiplicity 2 due to the case [ii] the common roots of $f=1$ (resp. $f=a$ ) and $g=a$ (resp. $g=1$ ).

On the other hand, the poles of the left hand side of (4.1) occur with multiplicity 2 due to the following two cases:

[iii] The 1- or $a$-points of $f$ (resp. $g$ ) which are neither 1- nor $a$-points of $g$ (resp. $f$ ),

[iv] the zeros of $f^{\prime}$ such that $f \neq 0,1, a$ or the zeros of $g^{\prime}$ such that $g \neq$ $0,1, a$, where the multiplicities of the zeros of $f^{\prime}$ and $g^{\prime}$ are different.

Hence we see that there are no points satisfying the above [i], [ii], [iii] or [iv], so that $f$ and $g$ share 1 and $a$ IM. Therefore by Theorem $\mathrm{F}, f$ and $g$ are connected with one of the relations stated in Theorem F. Further straightforward computations show that only two relations $(f-(1 / 2))(g-(1 / 2)) \equiv 1 / 4$ (with $a=1 / 2$ ) and $(f-1)(g-1) \equiv 1$ (with $a=2$ ) are suitable for $\phi_{6} \equiv 0$.

We next consider the case $a=-1$. In this case $\phi_{6} \equiv 0$ implies $\phi_{4} \equiv 0 . \quad \phi_{4} \equiv 0$ implies that any 1- and $a$-point of $f$ (resp. $g$ ) is a 1- or an $a$-point of $g$ (resp. $f$ ). The same argument as in the case 2.1 yields that $f$ and $g$ are connected with the relation with $a=-1$ stated in Theorem F, i.e., $f g \equiv 1$. But, a direct computation shows that this is not suitable for $\phi_{4} \equiv 0$.

\subsection{The case $\phi_{7} \equiv 0$}

The same reasoning as in the case 2.3 shows that only two relations $f+g \equiv 2$ (with $a=2$ ) and $f+g \equiv 1$ (with $a=1 / 2$ ) are suitable for $\phi_{7} \equiv 0$.

\subsection{The case $\phi_{8} \equiv 0$}

If $a=-1$, then $\phi_{8} \equiv \phi_{4}$. Since we have already handled the case $\phi_{4} \equiv 0$ with $a=-1$ in 2.3 , we may consider the case $a \neq-1$. First we easily see that $f$ and $g$ share $1 \mathrm{IM}$ by considering the residue of $\phi_{8}$ at any 1-point of $f$ or $g$, where we used the assumption $a \neq-1$. Next, we prove that $f$ and $g$ share $a$ IM $^{\prime \prime}$, i.e., $\bar{N}(r ; f=a, g \neq a)+\bar{N}(r ; g=a, f \neq a)=S(r)$. To show this, we suppose that $\bar{N}(r ; f=a, g \neq a)+\bar{N}(r ; g=a, f \neq a) \neq S(r)$, and will seek a contradiction. Under this assumption, we have $-1<a<0$. In fact, (without loss of generality) 
we may assume that $\bar{N}(r ; f=a, g \neq a) \neq S(r)$. From Lemma 2 we see that there exists a point $z_{a}$ satisfying $f\left(z_{a}\right)=a$ with multiplicity $p(\geqq k+1)$ and $g\left(z_{a}\right)=b(\neq a, 1,0, \infty)$ with multiplicity 1 . By the computation of the residue of $\phi_{8}$ at $z_{a}$ we have $p-1+a p=0$, i.e., $(a+1) p=1$, which gives $-1<a<0$. Further the same reasoning shows that if $\bar{N}(r ; f=a, g \neq a) \neq S(r)$, then any $a$-point of $f$ which is not an $a$-point of $g$ has multiplicity $\geqq(a+1)^{-1} \equiv p_{0}$ $(\geqq k+1 \geqq 13)$. In the same way, if $\bar{N}(r ; g=a, f \neq a) \neq S(r)$, then any $a$-point of $g$ which is not an $a$-point of $f$ has multiplicity $\geqq(a+1)^{-1} \equiv p_{0}$ ( $\geqq k+1 \geqq 13$ ). Hence (by taking the fact that $f$ and $g$ share 1 IM into account) in the same way as in $(3.3)^{\prime}$ in Lemma 3 we have

$$
\begin{aligned}
& m_{1, a}(r ; f, g)+2 \tilde{N}_{1, a}^{\prime}(r ; f, g)+3 \tilde{N}_{1, a}^{\prime \prime}(r ; f, g) \\
& \quad+p_{0}\{\bar{N}(r ; f=a, g \neq a)+\bar{N}(r ; g=a, f \neq a)\} \\
& \leqq 2\left\{\bar{N}(r, 0)+\bar{N}(r, \infty)+\tilde{N}_{1, a}(r ; f, g)\right\}+\bar{N}(r ; f=a, g \neq a) \\
&+\bar{N}(r ; g=a, f \neq a)-\left\{N_{1}^{\prime}(r, f)+N_{1}^{\prime}(r, g)\right\}+S(r)
\end{aligned}
$$

and so

$$
\begin{aligned}
& p_{0}\{\bar{N}(r ; f=a, g \neq a)+\bar{N}(r ; g=a, f \neq a)\} \leqq 2\{\bar{N}(r, 0)+\bar{N}(r, \infty)\} \\
& \quad+\{\bar{N}(r ; f=a, g \neq a)+\bar{N}(r ; g=a, f \neq a)\}+S(r) .
\end{aligned}
$$

If $z_{\beta}$ satisfies $f^{\prime}\left(z_{\beta}\right)=0, f\left(z_{\beta}\right) \neq 0,1, a$, (resp. $\left.g^{\prime}\left(z_{\beta}\right)=0, g\left(z_{\beta}\right) \neq 0,1, a\right)$ then $\phi_{8} \equiv 0$ implies that $g^{\prime}\left(z_{\beta}\right)=0, g\left(z_{\beta}\right) \neq 0,1$ (resp. $f^{\prime}\left(z_{\beta}\right)=0, f\left(z_{\beta}\right) \neq 0,1$ ). Hence by Lemma 2

$$
\bar{N}_{1}^{\prime}(r, f)+\bar{N}_{1}^{\prime}(r, g) \leqq 2 \tilde{N}\left(r ; f^{\prime}=g^{\prime}=0, f \neq 0, g \neq 0\right)=S(r) .
$$

In view of (3.13) we have

$$
\left.2\left\{N_{1}(r, 0)+N_{1}(r, \infty)\right\} \leqq \bar{N}(r ; f=a, g \neq a)+\bar{N}(r ; g=a, f \neq a)\right\}+S(r) .
$$

Since we have already considered the case $\phi_{6} \equiv 0$ in 2.3 and $\phi_{7} \equiv 0$ in 2.4 , we may now consider the case $\phi_{6} \not \equiv 0$ and $\phi_{7} \not \equiv 0$. Substituting (4.3) and (4.4) into (3.15) with $a \neq-1$, we obtain

$$
2\{\bar{N}(r, 0)+\bar{N}(r, \infty)\} \leqq 9\{\bar{N}(r ; f=a, g \neq a)+\bar{N}(r ; g=a, f \neq a)\}+S(r) .
$$

The combination of (4.2) and (4.5) gives $p_{0} \leqq 10$, which is a contradiction. This proves that $f$ and $g$ share $a \mathrm{IM}^{\prime \prime}$. Thus we deduce from Theorem $\mathrm{F}^{\prime}$ that $f$ and $g$ are connected with one of the relations with $a \neq-1$ stated in Theorem F. But straightforward computations show that none of the relations stated in Theorem $F$ are suitable for $\phi_{8} \equiv 0, \phi_{6} \not \equiv 0$ and $\phi_{7} \not \equiv 0$.

2.6. The case $\phi_{9} \equiv 0\left(, \phi_{6} \not \equiv 0, \phi_{7} \not \equiv 0\right)$

The same reasoning as in the case 2.5 shows that there is not a pair of $f$ and $g$ satisfying $\phi_{9} \equiv 0, \phi_{6} \not \equiv 0$ and $\phi_{7} \not \equiv 0$. 
2.7. The case $\phi_{2} \not \equiv 0, \phi_{3} \not \equiv 0, \phi_{6} \not \equiv 0, \phi_{7} \not \equiv 0, \phi_{8} \not \equiv 0, \phi_{9} \not \equiv 0$ have

First we consider the case $a \neq-1$. Combining (3.3), (3.15) and (3.13), we

$$
\tilde{N}_{1, a}^{\prime \prime}(r)+(k-15) \bar{N}_{1, a}(r) \leqq S(r) \text {. }
$$

On the other hand, using (3.3), (3.16) and (3.13) we have

$$
(k-7) \bar{N}_{1, a}(r) \leqq \tilde{N}_{1, a}^{\prime \prime}(r)+S(r) .
$$

Substituting (4.7) into (4.6), it follows that $(k-11) \bar{N}_{1, a}(r) \leqq S(r)$. Since $k \geqq 12$, this implies that $\bar{N}_{1, a}(r)=S(r)$, and so $\tilde{N}_{1, a}^{\prime \prime}(r)=S(r)$ by $(4.6)$.

Now assume that $a=-1$. Combining (3.3) and (3.14), we have

$$
(k-4) \bar{N}_{1, a}(r) \leqq 3 \tilde{N}_{1, a}^{\prime \prime}(r)+S(r) .
$$

On the other hand, we use (3.3), (3.15) and (3.13) to obtain

$$
\tilde{N}_{1, a}^{\prime \prime}(r)+(k-7) \bar{N}_{1, a}(r) \leqq S(r) .
$$

Taking the fact $k \geqq 7$ into account, we deduce from (4.8) and (4.9) that $\tilde{N}_{1, a}^{\prime \prime}(r)=$ $S(r)$ and $\bar{N}_{1, a}(r)=S(r)$.

Hence, $\bar{N}_{1, a}(r)=S(r)$ and $\tilde{N}_{1, a}^{\prime \prime}(r)=S(r)$ hold in both cases. From (3.13) and (3.14) we obtain $N(r, 0)+N(r, \infty)=S(r)$, and so by Lemma 1 and the second fundamental theorem $\bar{N}(r, 1, f), \bar{N}(r, a, f)=T(r, f)+S(r)$ and $\bar{N}(r, 1, g)$, $\bar{N}(r, a, g)=T(r, g)+S(r)$. On the other hand, $\bar{N}_{1, a}(r)=S(r)$ implies that $f$ and $g$ share two values 1 and $a \mathrm{IM}^{\prime \prime}$, and so we deduce from Theorem $\mathrm{F}^{\prime}$ that $f$ and $g$ are connected with one of the relations in Theorem F. Therefore we obtain $f g \equiv 1$ with $a=-1$ in this case.

This completes the proof of Theorems 1 and 2 .

Remark 1. The author does not know whether Theorem 1 holds for positive integers $k(3 \leqq k \leqq 11)$ or not.

Remark 2. The author does not know whether Theorem 2 holds for positive integers $k(3 \leqq k \leqq 6)$ or not.

Acknowledgement. The author is very grateful to the referee for his (or her) valuable comments.

\section{REFERENCES}

[ 1] S. S. Bhoosnurmath AND H. S. Gopalakrishna, Uniqueness theorems for meromorphic functions, Math. Scand., 39 (1976), 125-130.

[2] G. G. Gundersen, Meromorphic functions that share four values, Trans. Amer. Math. Soc., 277 (1983), 545-567

[3] W K. Hayman, Meromorphic Functions, Clarendon Press, Oxford, 1964.

[4] E. MuEs, Meromorphic functions sharıng four values, Complex Variables Theory Appl., 12 (1989), 169-179. 
[5] R. Nevanlinna, Le théorème de Picard-Borel et la théorie des fonctions méromorphes, Parıs, Gauthier Villars, 1929.

[6] H. UEDA, Unicity theorems for meromorphic or entıre functions, Koda1 Math. J., 3 (1980), 457-471.

[7] H. UEDA, Unicity theorems for meromorphic functions sharing five or six values in some sense, Koda1 Math. J., 18 (1995), 494-505.

Department of Mathematics

Daido Institute of TeChNOLOGY

DAIDO, Minami, NAGOYa 457-8530

JAPAN 